\title{
ZERO-CYCLES ON SELF-PRODUCT OF MODULAR CURVES
}

\author{
Kenichiro KimURA
}

$\S 1$ Introduction. Let $X$ be a projective smooth variety over $\mathbb{Q}$. Let $S$ be a finite set of primes such that $X$ has a projective smooth model $\mathcal{X}$ over $U:=\operatorname{spec} \mathbb{Z}\left[\frac{1}{S}\right]$. Then from the works of Bloch([Bl1]) and Sherman, we have the following exact localization sequence in algebraic $K$-theory, using Gersten's conjecture for $\mathcal{K}_{2}$ in a mixed characteristic setting that was proven by Bloch $([\mathrm{Bl}])$

$$
H^{1}\left(\mathcal{X}, \mathcal{K}_{2}\right) \rightarrow H^{1}\left(X, \mathcal{K}_{2}\right) \stackrel{\partial}{\rightarrow} \underset{p \in U}{\oplus} \operatorname{Pic}\left(X_{p}\right) \rightarrow C H^{2}(\mathcal{X}) \rightarrow C H^{2}(X) \rightarrow 0
$$

where $X_{p}$ is the fiber of $\mathcal{X}$ at the prime $p$.

A conjecture of Beilinson on the special values of $L$-function and Tate conjecture tell us that the cokernel of $\partial$ is torsion. See the remark after Theorem 2.5 in [Lang] for an account of this.

In this paper we present a modest evidence for this conjecture. We consider the case where $X$ is a self-product of a curve $C$ which is modular in the sense defined below. We make use of elements in the $K$-cohomology group $H^{1}\left(X, \mathcal{K}_{2}\right)$ which are constructed by Flach $([\mathrm{Fl}])$ and Mildenhall([Mi]). Our main result (theorem 2.3) is that the boundary map

$$
\partial: H^{1}\left(C \times C, \mathcal{K}_{2}\right) \rightarrow \bigoplus_{p \in \tilde{S}} \operatorname{Pic}(C \times C \bmod p)
$$

has a torsion cokernel. Here $\tilde{S}$ is a set of primes which satisfy certain conditions explained below. By using an idea of Murty and Baba in [BaMu] and results by $\operatorname{Ribet}([\mathrm{Ri}])$ and Momose $([\mathrm{Mo}])$ on the image of the Galois representaion associated to $C$, it is shown in the proposition 3.1 that under certain mild assumption on $C$ the set $\tilde{S}$ has density 1 .

When $C$ is an elliptic curve, $\tilde{S}$ contains all but finite number of primes. By using this fact, Langer and Saito give a finiteness result for the torsion subgroup of the Chow group $C H^{2}(C \times C)([\mathrm{LS}])$. When genus $(C)>1$ this is still not known. So the finiteness result we obtain is about $C H^{2}\left(C \times C \times \operatorname{Spec} \mathbb{Q}_{p}\right)$ for the primes $p \in \tilde{S}$. The conditions on the primes are almost equivalent to irreducibility of the characteristic polynomial of the Hecke operator $T_{p}$ on $\Gamma\left(C, \Omega_{C}^{1}\right)$. In the appendix we discuss the relation of this condition with Maeda's conjecture ([HiMa],[BaMu]) on irreducibility of Hecke polynomials.

Th author thanks Shuji Saito for discussions and Andreas Langer for careful reading of earlier version of this paper and correcting some errors. 
$\S 2$ The result. For an abelian group $M, M_{\mathbb{Q}}$ denotes $M \otimes \mathbb{Q}$.

Let $f$ be an element of $S_{2}\left(\Gamma_{0}(N)\right)$ or $S_{2}\left(\Gamma_{1}(N)\right)$ for some integer $N$ which is an eigenfunction of the Hecke operators $T_{n}$ for all $n>0$, and let $f \mid T_{n}=a_{n} f$. Let $K$ be the subfield of $\mathbb{C}$ generated over $\mathbb{Q}$ by $a_{n}$ for all $n$. Then by the Theorem $7.14 \mathrm{in}$ [Sh] there exists a unique abelian subvariety $A$ of $J_{0}(N)$ or $J_{1}(N)$ depending on whether $f \in S_{2}\left(\Gamma_{0}(N)\right)$ or $f \in S_{2}\left(\Gamma_{1}(N)\right)$ which is defined over $\mathbb{Q}$ with a homomorphism $\theta: K \rightarrow \operatorname{End}_{\mathbb{Q}}(A) \otimes \mathbb{Q}$ which has the following properties:

(1) $\operatorname{dim} A=[K: \mathbb{Q}]$.

(2) $\theta\left(a_{n}\right)$ is the restriction of $T(n)$ to $A$.

Let $C$ be a projective smooth curve over $\mathbb{Q}$.

Definition. The curve $C$ is modular if the jacobian $J a c(C)$ of $C$ is isogenous over $\mathbb{Q}$ to an abelian variety $A$ which is associated with a modular form $f$ in $S_{2}\left(\Gamma_{0}(N)\right)$ or $S_{2}\left(\Gamma_{1}(N)\right)$ for some $N$ in the above sense.

For example the curves $X_{0}(23)$ and $X_{1}(13)$ are modular in this sense. Assume that $C$ is modular. Then there is an algebraic number field $K$ with $[K$ : $\mathbb{Q}]=g=\operatorname{genus}(C)$ and a homomorphism $\theta: K \rightarrow \operatorname{End}_{\mathbb{Q}}(\operatorname{Jac}(C)) \otimes \mathbb{Q}$. We identify $K$ with $\theta(K)$. In the following we denote by $X$ the modular curve $X_{0}(N)$ or $X_{1}(N)$ depending on whether $C$ is modular for $\Gamma_{0}(N)$ or $\Gamma_{1}(N)$. If $C$ is modular for $\Gamma_{1}(N)$ we assume that the modular form $f$ associated to $C$ satisfies the condition (7.5.9) in [Sh] i.e. the field $K$ is generated by $a_{n}$ for all $n$ prime to $N$.

There is a finite set of primes $S$ which contains all the primes dividing $N$ such that there are projective smooth models $\mathcal{X}$ and $\mathcal{C}$ over Spec $\mathbb{Z}\left[\frac{1}{S}\right]$ of $X$ and $C$ respectively. There is also an algebraic correspondence $\Gamma \subset \mathcal{X} \times \mathcal{C}$ which is finite over $\mathcal{C}$ such that the generic fiber $\Gamma_{\mathbb{Q}}$ of $\Gamma$ induces a surjective morphism $\Gamma_{\mathbb{Q}_{*}}: \operatorname{Jac}(X) \rightarrow \operatorname{Jac}(C)$. For a scheme $\mathcal{Y}$ over Spec $\mathbb{Z}\left[\frac{1}{S}\right]$ and for a prime $p \notin S Y_{p}$ denotes $\mathcal{Y} \times \times_{\text {Spec } \mathbb{Z}\left[\frac{1}{S}\right]} \operatorname{Spec} \mathbb{F}_{p}$. Let $p \notin S$ be a prime. First we consider the case where $X=X_{0}(N)$. Let $\mathcal{X}_{0}(N p)$ be the modular curve over Spec $\mathbb{Z}\left[\frac{1}{S}\right]$ classifying pairs $(E, G)$ of elliptic curves and cyclic subgroups of $E$ of order $N p$. The Hecke correspondence $T(p) \subset \mathcal{X} \times \mathcal{X}$ is the image of $\mathcal{X}_{0}(N p)$ under the map $(E, G) \mapsto(E, p G) \times\left(E / G^{\prime}, G / G^{\prime}\right)$. Here $G^{\prime}$ denotes the subgroup of $G$ of order $p$. By ([Sh], Corollary 7.10) The divisor $T(p)_{p}$ on $X_{p} \times X_{p}$ is the sum of the graph of Frobenius endomorphism and its transpose. Since the composition of correspondences $\Gamma \circ{ }^{t} \Gamma=(\operatorname{deg} \Gamma) \Delta \subset \mathcal{C} \times \mathcal{C}$ where $\operatorname{deg} \Gamma$ is the degree of $\Gamma$ over $\mathcal{C}$, it follows that the correspondence

$$
\left(\Gamma \circ T(p) \circ{ }^{t} \Gamma\right)_{p}=\operatorname{deg} \Gamma\left(\Gamma_{F}+{ }^{t} \Gamma_{F}\right)
$$

where $\Gamma_{F}$ is the graph of frobenius endomorphism $F$ on $C_{p}$. Thus we see that there is the equality

$$
(\operatorname{deg} \Gamma)\left(F^{2}-a_{p} F+p\right)=0
$$

in $\operatorname{End}_{\mathbb{F}_{p}}\left(\operatorname{Jac}\left(C_{p}\right)\right)_{\mathbb{Q}}$.

Next we consider the case where $X=X_{1}(N)$. Let $\mathcal{X}_{1}(N ; p)$ be the modular curve over Spec $\mathbb{Z}\left[\frac{1}{S}\right]$ classifying triples $(E, P, G)$ of elliptic curves, points of exact order 
$N$ and cyclic subgroups of order $p$. The Hecke correspondence $T(p)$ on $\mathcal{X} \times \mathcal{X}$ is the image of $\mathcal{X}_{1}(N ; p)$ by the map $(E, P, G) \mapsto(E, P) \times(E / G, P)$. Then again by ([Sh], Corollary 7.10) the divisor $T(p)_{p}$ on $X_{p} \times X_{p}$ is equal to $\Gamma_{F r}+\Gamma_{<p>}{ }^{t} \Gamma_{F r}$ where $\langle p\rangle: \mathcal{X} \rightarrow \mathcal{X}$ is the map $(E, P) \mapsto(E, p P)$. We have the equality

$$
\left(\Gamma_{p} \circ T(p) \circ{ }^{t} \Gamma\right)_{p}=(\operatorname{deg} \Gamma) \Gamma_{F}+\left(\Gamma \circ \Gamma_{<p>} \circ{ }^{t} \Gamma\right)_{p} \circ{ }^{t} \Gamma_{F} .
$$

We denote by $\langle p\rangle^{\prime}$ the endomorphism of $\operatorname{Jac}\left(C_{p}\right)$ induced by the correspondence $\left(\Gamma \circ \Gamma_{<p>} \circ^{t} \Gamma\right)_{p}$. Since the morphism $<p>$ is rational over $\mathbb{Q}$ we see that there is the equality

$$
(\operatorname{deg} \Gamma)\left(F^{2}-a_{p} F\right)+p<p>^{\prime}=0
$$

in $\operatorname{End}_{\mathbb{F}_{p}}\left(\operatorname{Jac}\left(C_{p}\right)\right)_{\mathbb{Q}}$.

We know by Flach, Mildenhall([Fl],[Mi]) in the case $X=X_{0}(N)$ and Weston([We] $)$ in the case $X=X_{1}(N)$ that there is an element $\alpha_{p} \in H^{1}\left(X \times X, \mathcal{K}_{2}\right)_{\mathbb{Q}}$ such that $\partial\left(\alpha_{p}\right)=\Gamma_{F r}$ the graph of Frobenius endomorphism. Here the map

$$
\partial: H^{1}\left(X \times X, \mathcal{K}_{2}\right) \rightarrow \bigoplus_{p \notin S} \operatorname{Pic}\left(X_{p} \times_{\mathbb{F}_{p}} X_{p}\right)
$$

is the boundary map arising from the localization sequence in algebraic $K$-theory. For a smooth projective variety $V$ over a field $k$, The group $H^{n-1}\left(V, \mathcal{K}_{n}\right)$ is isomorphic to the higher Chow group $C H^{n}(V, 1)([$ Land],[Mü]).

We define the composition $\Gamma \circ \alpha_{p} \in C H^{2}(X \times C, 1)$ to be $p r_{13 *}\left(p r_{12}^{*} \alpha_{p} \cdot p r_{23}^{*} \Gamma\right)$ where $p r_{i j}$ are the projections on $X \times X \times C$.

Lemma 2.1. We have the equality

$$
\partial\left(\Gamma \circ \alpha_{p} \circ{ }^{t} \Gamma\right)=(\operatorname{deg} \Gamma) \Gamma_{F}
$$

Proof. We have the following commutative diagrams:

$$
\begin{aligned}
& C H^{2}(X \times X \times C, 1)_{\mathbb{Q}} \stackrel{\partial}{\longrightarrow} C H^{1}\left(X_{p} \times X_{p} \times C_{p}\right)_{\mathbb{Q}} \\
& p r_{23}^{*} \Gamma_{\mathbb{Q}} \cdot \downarrow \quad p r_{23}^{*} \Gamma_{p} \cdot \downarrow \\
& C H^{3}(X \times X \times C, 1)_{\mathbb{Q}} \stackrel{\partial}{\longrightarrow} C H^{2}\left(X_{p} \times X_{p} \times C_{p}\right)_{\mathbb{Q}} \\
& C H^{3}(X \times X \times C, 1)_{\mathbb{Q}} \stackrel{\partial}{\longrightarrow} C H^{2}\left(X_{p} \times X_{p} \times C_{p}\right)_{\mathbb{Q}} \\
& p r_{13 *} \downarrow \quad p r_{13 *} \downarrow \\
& C H^{2}(X \times C, 1)_{\mathbb{Q}} \stackrel{\partial}{\longrightarrow} C H^{1}\left(X_{p} \times C_{p}\right)_{\mathbb{Q}}
\end{aligned}
$$

Commutativity of the first diagram follows from the compatibility of the boundary map $\partial$ with $K_{0}\left(\mathcal{X}^{2} \times \mathcal{C}\right)$ module structure on algebraic $K$-theory. Here $\mathcal{C}$ resp. $\mathcal{X}$ 
is a projective smooth model of $C$ resp. $X$ over $\operatorname{Spec} \mathbb{Z}_{(p)}$. See $([\mathrm{Qu}], \S 3)$ for the definition of $K_{0}\left(\mathcal{X}^{2} \times \mathcal{C}\right)$ module structure. Commutativity of the second diagram follows from the functoriality of the map $\partial$ on algebraic $K$-theory with respect to the push-forward by projective morphisms. See ([Sri], (5.11)) for the definition of the push-forward.

Hence there is the equality

$$
\begin{aligned}
& \Gamma_{p} \circ \Gamma_{F r} \\
= & p r_{13 *}\left(p r_{23}^{*} \Gamma_{p} \cdot \partial\left(p r_{12}^{*} \alpha_{p}\right)\right) \\
= & p r_{13 *}\left(\partial\left(p r_{23}^{*} \Gamma_{\mathbb{Q}} \cdot p r_{12}^{*} \alpha_{p}\right)\right) \\
= & \partial\left(p r_{13 *}\left(p r_{23}^{*} \Gamma_{\mathbb{Q}} \cdot p r_{12}^{*} \alpha_{p}\right)\right) .
\end{aligned}
$$

The same argument for composition with ${ }^{t} \Gamma$ concludes the proof.

By the same argument we also obtain

Lemma 2.2. Assume that $\operatorname{deg} \Gamma \neq 0$. Then for an element $a \in K \subset \operatorname{End}(\operatorname{Jac}(C))_{\mathbb{Q}}$ the composition $a \circ \Gamma_{F} \in \operatorname{Pic}\left(C_{p} \times C_{p}\right)_{\mathbb{Q}}$ is contained in $\operatorname{Im}(\partial)_{\mathbb{Q}}$. Here we identify $\operatorname{End}(\operatorname{Jac}(C))_{\mathbb{Q}} \subset \operatorname{End}\left(\operatorname{Jac}\left(C_{p}\right)\right)_{\mathbb{Q}}$ with a subspace of $\operatorname{Pic}\left(C_{p} \times C_{p}\right)_{\mathbb{Q}}$.

From the lemma we see that if $\operatorname{deg} \Gamma \neq 0$ then $\operatorname{Im}(\partial)_{\mathbb{Q}}$ contains the subring of $\operatorname{End}\left(\operatorname{Jac}\left(C_{p}\right)\right) \otimes \mathbb{Q}$ which is generated by $F$ and $K$.

Since the modular form $f$ associated with $C$ is a Hecke eigenfunction for all $T_{n}$, by the Proposition 3.53 in [Sh] $f$ is in $S_{2}\left(\Gamma_{0}(N), \psi\right)$ for a unique character $\psi:(\mathbb{Z} / N \mathbb{Z})^{*} \rightarrow K^{*}$. Note that $\psi$ is trivial if $f \in S_{2}\left(\Gamma_{0}(N)\right)$.

As we assume that the condition (7.5.9) in [Sh] holds, By the theorems 7.14 and 7.16 in loc.cit. it follows that the characteristic polynomial of the action of Frobenius endomorphism on the Tate module $T_{l}(\operatorname{Jac}(C))$ is equal to

$$
\prod_{\sigma: K \hookrightarrow \mathbb{C}}\left(T^{2}-a_{p}^{\sigma} T+\psi(p)^{\sigma} p\right) .
$$

If this polynomial is separable the dimension the commutator of $F$ in $\operatorname{End}_{\mathbb{Q}_{l}}\left(T_{l}(\operatorname{Jac}(C))\right)_{\mathbb{Q}}$ is equal to $2 \operatorname{genus}(C)$.

Theorem 2.3. Assume that $\operatorname{deg} \Gamma \neq 0$. Then the cokernel of the map

$$
\partial: H^{1}\left(C \times C, \mathcal{K}_{2}\right) \rightarrow \bigoplus_{p} \operatorname{Pic}\left(C_{p} \times_{\mathbb{F}_{p}} C_{p}\right)
$$

is torsion with $p$ running over the set of primes which satisfy the following condition: (1) $p \notin S$. (2) $F \notin K$. (3) The polynomial (4) is separable.

Proof. By the Theorem 12.5 in [Mil] the map

$$
\operatorname{End}_{\mathbb{F}_{p}}\left(J a c\left(C_{p}\right)\right) \otimes \mathbb{Q}_{l} \rightarrow \operatorname{End}_{\mathbb{Q}_{l}}\left(T_{l}\left(J a c\left(C_{p}\right)\right)_{\mathbb{Q}}\right)
$$


is injective. So the dimension over $\mathbb{Q}_{l}$ of the subring of $\operatorname{End}_{\mathbb{Q}_{l}}\left(T_{l}\left(J a c\left(C_{p}\right)_{\mathbb{Q}}\right)\right)$ generated by $F$ and $K$ over $\mathbb{Q}_{l}$ is $2 g$. It follows that the subspace $\operatorname{End}_{\mathbb{F}_{p}}\left(\operatorname{Jac}\left(C_{p}\right)\right)_{\mathbb{Q}} \subset$ $\operatorname{Pic}\left(C_{p} \times_{\mathbb{F}_{p}} C_{p}\right) \otimes \mathbb{Q}$ is contained in $\operatorname{Im}(\partial)_{\mathbb{Q}}$. The assertion of the theorem follows from the equality

$$
\operatorname{Pic}\left(C_{p} \times_{\mathbb{F}_{p}} C_{p}\right) \otimes \mathbb{Q}=p r_{1}^{*} \operatorname{Pic}\left(C_{p}\right)_{\mathbb{Q}} \oplus \operatorname{End}_{\mathbb{F}_{p}}\left(J a c\left(C_{p}\right)\right)_{\mathbb{Q}} \oplus p r_{2}^{*} \operatorname{Pic}\left(C_{p}\right)_{\mathbb{Q}} .
$$

Remark. When $C$ is modular for $\Gamma_{0}(N)$ and the field $K$ is totally real the conditions (2) and (3) are satisfied if $a_{p}^{\tau} \neq a_{p}^{\sigma}$ for any two distinct embeddings $\sigma$ and $\tau$ of $K$ into $\mathbb{R}$ and $\sqrt{p} \notin K$.

Corollary 2.4. If a prime $p$ satisfies the conditions (1), (2) and (3), the non-pprimary torsion part of $C H^{2}\left((C \times C) \times \mathbb{Q}_{\mathbb{Q}}\right)$ is finite.

Proof. From the works of Bloch $([\mathrm{Bl}]])$ and Sherman, we have the following exact localization sequence in algebraic $K$-theory, using Gersten's conjecture for $K_{2}$ in a mixed characteristic setting that was proven by Bloch $([\mathrm{Bl}])$

$$
H^{1}\left(\mathcal{Y}, \mathcal{K}_{2}\right) \rightarrow H^{1}\left(Y, \mathcal{K}_{2}\right)=C H^{2}(Y, 1) \stackrel{\partial}{\rightarrow} \operatorname{Pic}\left(Y_{p}\right) \rightarrow C H^{2}(\mathcal{Y}) \rightarrow C H^{2}(Y) \rightarrow 0
$$

Here $Y=(C \times C) \times_{\mathbb{Q}} \mathbb{Q}_{p}, \mathcal{Y}$ is a projective smooth model of $Y$ over Spec $\mathbb{Z}_{p}$ and $Y_{p}=\mathcal{Y} \bmod p$. By the argument of Raskind in [Ra, Theorem 1.9], the reduction map

$$
C H^{2}(\mathcal{Y})\{l\} \rightarrow C H^{2}\left(Y_{p}\right)\{l\}
$$

is injective for all primes $l \neq p$. Here $\{l\}$ means the $l$-primary torsion part. Since $C H^{2}\left(Y_{p}\right)_{\text {tors }}$ is finite $([\mathrm{CTSS}]$, Theorem 1$)$ the corollary follows.

$\S 3$ About the condition (3). In this section we consider the condition (3) in the theorem 2.3. We assume that the curve $C$ is modular for $\Gamma_{0}(N)$ for some integer $N$. In this case the field $K$ is totally real. Suppose that $J(C)$ satisfies the following condition: $\operatorname{End}(J(C) \otimes \overline{\mathbb{Q}}) \otimes \mathbb{Q}=K$.

Proposition 3.1. Under the above assumption, we have the inequality

$$
\#\left\{p \leqq x \mid a_{p} \text { is in a proper subfield of } K\right\} \leqq c \frac{x}{(\log x)^{1+\delta}}
$$

for some constants $c$ and $\delta>0$.

Proof. The proof follows the same argument as in $[\mathrm{BaMu}]$. We sketch the outline. Let $L \subset K$ be a proper subfield. Let $\lambda \subset \mathcal{O}_{K}$ be a prime of degree $d \geqq 2$ over $\mathcal{L}=\lambda \cap \mathcal{O}_{L}$. Let

$$
\rho_{f, \lambda}: \operatorname{Gal}(\overline{\mathbb{Q}} / \mathbb{Q}) \rightarrow G L_{2}\left(\mathcal{O}_{K} / \lambda\right)
$$

be the Galois representaion $T_{l}(J(C)) \otimes_{\mathcal{O}_{K}} \mathcal{O}_{K} / \lambda$ where the prime $l$ is the characteristic of $\mathcal{O}_{K} / \lambda$. The representaion $\rho_{f, \lambda}$ has the property that for any prime 
$p \nmid l N$, we have the equality $\operatorname{trace}\left(\rho_{f, \lambda}\left(F r o b_{p}\right)\right)=a_{p}$. It follows from the proof of the theorem 4.2 in $[\mathrm{Mo}]$ that the image of $\operatorname{Gal}(\overline{\mathbb{Q}} / \mathbb{Q})$ in $G L_{2}\left(\mathcal{O}_{K} / \lambda\right)=H_{\lambda}$ for almost all rational primes $l$ where $H_{\lambda}:=\left\{u \in G L_{2}\left(\mathcal{O}_{K} / \lambda\right) \mid \operatorname{det} u \in \mathbb{F}_{l}^{*}\right\}$. Note that under our assumtion the field $F$ in loc.cit.is $K$, the field $L_{f}$ is $\mathbb{Q}$ and the algebra $E=E_{f}=\operatorname{End}_{K}\left(H_{1}(J(C) \times \mathbb{C}, \mathbb{Q})\right)$.

Let $\mathcal{S} \subset H_{\lambda}$ be the set of elements whose trace lies in the subfield $\mathcal{O}_{L} / \mathcal{L} \subset \mathcal{O}_{K} / \lambda$. Then by a counting argument we see that

$$
\# \mathcal{S} \leqq e_{1} N_{\mathcal{L}}^{2 d+1+1 / d_{2}} \quad \text { and } \quad \# H_{\lambda}=N_{\mathcal{L}}^{3 d+1 / d_{2}}
$$

for a positive constant $e_{1}$. Here $d_{2}=\left[\mathcal{O}_{L} / \mathcal{L}: \mathbb{F}_{l}\right]$.

Let $M$ be the fixed field of the kernel of the representation $\rho_{f, \lambda}$. By an effective version of the Chebotarev density theorem in $M$, there exist absolute constants $c_{1}$ and $c_{2}$ such that if $x \geqq 3$ and $\log x \geqq c_{1}\left(\log d_{M}\right)\left(\log \log d_{M}\right)\left(\log \log \log 6 d_{M}\right)$, then there is the inequality

$$
\#\left\{p \leqq x \mid \operatorname{trace}\left(\rho_{f, \lambda}\left(\text { Frob }_{p}\right)\right) \in \mathcal{O}_{L} / \mathcal{L}\right\} \leqq c_{2} \frac{\#(\mathcal{S})}{\#\left(H_{\lambda}\right)} \frac{x}{\log x}
$$

Here $d_{M}$ is the discriminant of the field $M$. Since we have the bound due to Hensel

$$
\log d_{M} \leqq\left(n_{M}-1\right) \sum_{p \in P_{L}} \log p+n_{M} \log n_{M}
$$

where $n_{M}=[M: \mathbb{Q}]$ and $P_{L}$ is the set of rational primes which ramify in $L$, we can choose such $\lambda$ that satisfies the inequality

$$
l \geqq c_{3}(\log x)^{\delta}
$$

for positive constants $c_{3}$ and $\delta$. Hence we obtain the bound

$$
\#\left\{p \leqq x \mid \operatorname{trace}\left(\rho_{f, \lambda}\left(F_{r o b}\right)\right) \in \mathcal{O}_{L} / \mathcal{L}\right\} \leqq c_{4} \frac{x}{(\log x)^{1+\delta}}
$$

for a constant $c_{4}$. Since there are only finitely many proper subfields of $K$, this finishes the proof.

Corollary 3.2. Under the same assumption as in the proposition the set of primes which satisfy the conditions (1), (2) and (3) in the theorem 2.3 has density 1.

Appendix. We continue to suppose that $C$ is modular for $\Gamma_{0}(N)$. The following is a table of the genus of the curves $X_{0}(N)$ and the numbers of the primes $p$ such that $p \nmid N, p<10000$ and which do not satisfy the condition (3) for $X_{0}(N)$. The curves $X_{0}(N)$ for $N=23,41,59$ are modular in our sense.

\begin{tabular}{|c|c|c|}
\hline$N$ & genus & number of primes \\
\hline 23 & 2 & 45 \\
41 & 3 & 1 \\
59 & 5 & 0 \\
\hline
\end{tabular}


The one prime for $N=41$ is 17 . We used a computer program in [St] to compute the characteristic polynomials of the Hecke operators. It seems that as the genus of the curve grows, the primes which do not satisfy the condition (3) become rarer very quickly. We will give a probabilistic argument about reason for the rareness of the primes which do not satisfy the condition (3). Let $S^{\prime}$ be the set of primes which do not satisfy the condition (3). We denote by $R$ the ring $K \cap \operatorname{End}(J(C))$. Let $n$ be an integer which is prime to the discriminant $d_{K}$ of $K$ and the conductor $F$ of $R$. Let $\mathcal{M} / \operatorname{Spec} \mathbb{Z}\left[\frac{1}{d_{K} F n}\right]$ be the moduli space of abelian schemes of relative dimension genus $(C)$ with level $n$ structure which have real multiplication by $R$. Take a number field $H$ so that $J(C)_{H}=J(C) \times_{\mathbb{Q}} H$ defines a section $s_{C} \in \mathcal{M}(U)$ for an open set $U$ in $\operatorname{Spec} \mathcal{O}_{H}$. For a prime $p \in S^{\prime}$ the field $K^{\prime}:=\mathbb{Q}\left(a_{p}\right)$ is a proper subfield of $K$. Then the characteristic polynomial of $F r o b_{p}$ on $T_{l}(J(C))$ is equal to

$$
\left(\prod_{\sigma: K^{\prime} \hookrightarrow \mathbb{R}}\left(T^{2}-a_{p}^{\sigma} T+p\right)\right)^{n}
$$

where $n=\left[K: K^{\prime}\right]$. This means that there is an isogeny

$$
J(C)_{p} \rightarrow\left(A^{\prime}\right)^{n}
$$

for an abelian subvariety $A^{\prime}$ of $J(C)_{p}$ of dimension $d=\left[K^{\prime}: \mathbb{Q}\right]$ which has a real multiplication by the ring of integers $\mathcal{O}_{K^{\prime}}$ of $K^{\prime}$. Let $\mathcal{L}$ be the subscheme of $\mathcal{M}$ which corresponds to the abelian schemes which are isogenous to the power of abelian subschemes with real multiplication by orders in proper subfields of $K$. The set $S^{\prime}$ is the image in $\operatorname{Spec} \mathbb{Z}\left[\frac{1}{S}\right]$ of $s_{C} \cap \mathcal{L}$. The dimension of $\mathcal{M}=\operatorname{genus}(\mathrm{C})+1$ and the codimentions of (infinitely many) irreducible components of $\mathcal{L}$ are at least genus $(\mathrm{C}) / 2$. So as the genus of $C$ grows, the probability that $s_{C}$ intersects $\mathcal{L}$ becomes smaller.

About irredcibility of Hecke polynomials, there is a conjecture called Maeda's conjecture which states that the characteristic polynomial of the $n$-th Hecke operator $T_{n}$ on $S_{k}\left(S L_{2}(\mathbb{Z})\right)$ is irreducible with Galois group $S_{d}$ for every $n$ ([BaMu], $[\mathrm{HiMa}])$. We raise a question which is somewhat similar to this conjecture:

Question. Is it true that when the genus of the curve $C$ is large, then the characteristic polynomial of the p-th Hecke operator $T_{p}$ on $\Gamma\left(C, \Omega_{C}^{1}\right)$ is irreducible for all but finitely many primes $p$ ?

If this question has an affirmative answer, then the condition (3) is satisfied by almost all primes, and it follows that for all but finitely many rational primes $p$ the $p$-primary torsion part of $C^{2}(C \times C)$ is cofinitely generated(cf. [La2], p278).

\section{REFERENCES}

[BaMu] Baba, S., Murty, R., Irreducibility of Hecke polynomials, Math.Res.Letters 10 (2003), 709-715.

[Bl1] Bloch, S., Lectures on Algebraic Cycles, Duke University Press, 1980.

[Bl2] Bloch, S., "A note on Gersten's conjecture in the mixed characteristic case" in Applications of Algebraic K-theory to Algebraic Geometry and Number Theory, Part I, II, Contemp. Math. 55 (1986), Amer. Math. Soc. Providence. 
[CTSS] Colliot-Thélène, J., Sansuc, J., Soulé, C., Torsion dans le groupe de Chow de codimension deux, Duke Math. J. 50 (1983), 763-801.

[Fl] Flach, M., A finiteness theorem for the symmetric square of an elliptic curve, Invent. Math 109 (1992), 307-327.

[HiMa] Hida, H., Maeda, Y., Non-abelian base change for totally real fields, Pacific J. Math. Special issue (1997), 189-217.

[Land] Landsburg, S. E., Relative Chow groups, Illinois J. Math. 35 (1991), 618-641.

[Lang] Langer, A., Zero cycles on Hibert-Blumenthal surfaces, Duke Math. J 103 (2000), 131163.

[La2] Langer, A., Finiteness of torsion in the codimension-two Chow group: an axiomatic approach., NATO Sci. Ser. C Math. Phys. Sci. 548 (2000), 277-284.

[LS] Langer, A., Saito, S., Torsion zero-cycles on the self-product of a modular elliptic curve, Duke Math. J 85 (1996), 315-357.

[Mi] Mildenhall, S., Cycles in a product of elliptic curves, and a group analogous to the class group, Duke Math. J 67 (1992), 387-406.

[Mil] Milne, J. S., Abelian varieties, Arithmetic geometry (Storrs, Conn., 1984), Springer, 1986, pp. 103-150.

[Mü] Müller-Stach, S., Constructing indecomposable motivic cohomology classes on algebraic surfaces, J. Algebraic Geom. 6 (1997), 513-543.

[Qu] Quillen, D., "Higher algebraic K-theory. I."in Proc. Conf., Battelle Memorial Inst., Seattle, Wash., 1972, Lecture Notes in Math. 341 (1973), 85-147.

[Ra] Raskind, W., "Torsion algebraic cycles on varieties over local fields" in Algebraic Ktheory: connections with geometry and topology (Lake Louise, AB, 1987), NATO Adv. Sci. Inst. Ser. C Math. Phys. Sci. 279 (1989), 343-388.

[Ri] Ribet, K.A., Galois representations attached to eigenforms with nebentypes, SLN 601 (1976), 17-52.

[Sh] Shimura, G., Introduction to the arithmetic theory of automorphic functions. Kano Memorial Lectures, No. 1. Publications of the Mathematical Society of Japan, No. 11., Iwanami Shoten, 1971.

[Sri] Srinivas, V., Algebraic K-theory, Progress in Math., vol. 90, Birkhäuser, 1991.

[St] Wetherell, J.L., Stein, W., Bernardi, D., Perrin-Riou, B., http://modular.fas.harvard.edu/Tables/heckegp.html.

[We] Weston,T., Algebraic cycles, modular forms and Euler systems, J. Reine Angew. Math. 543 (2002), 103-145.

Kenichiro Kimura

Institute of Mathematics

University of Tsukuba

Tsukuba, Ibaraki

305-8571

Japan

email: kimurak@math.tsukuba.ac.jp 